\title{
Fast and Low-dose Electron Ptychography
}

Jiamei Song ${ }^{1}$, Biying Song ${ }^{1}$, Liqi Zou ${ }^{1}$, Christopher Allen ${ }^{2,3}$, Hidetaka Sawada ${ }^{4}$, Fucai Zhang ${ }^{5}$, Xiaoqing Pan $^{6}$, Angus. I. Kirkland ${ }^{2,3}$, and Peng Wang ${ }^{1}$

1. National Laboratory of Solid State Microstructures, College of Engineering and Applied Sciences and Collaborative Innovation Center of Advanced Microstructures, Nanjing University, Nanjing, People's Republic of China.

2. Department of Materials, University of Oxford, Parks Road, Oxford, UK.

3. Electron Physical Sciences Imaging Centre, Diamond Lightsource Ltd., Diamond House, U.K.

4. JEOL Ltd, Akishima, Tokyo, Japan.

5. Department of Electrical and Electronic Engineering, Southern University of Science and Technology, Shenzhen, China.

6. Department of Chemical Engineering and Materials Science and Department of Physics and Astronomy, University of California, Irvine, CA, USA.

In recent years, there have been many significant developments made in scanning transmission electron microscopy (STEM), notably the development of aberration correctors and complementary electron optical components such as monochromators and high brightness guns [1]. These advances have made it possible to obtain a $0.5 \AA$ resolution at $300 \mathrm{kV}$ for radiation resistant materials. However, spatial resolution is still limited for beam-sensitive specimen such as organics, biological specimens, zeolites and ceramics due to radiation damage. Beam-sensitive specimens have varying tolerances to electron dose due to different damage mechanisms. Although it is possible to reduce the dose in the STEM geometry by decreasing the pixel dwell time or the probe current density, a large pixel array is still needed for atomic resolution imaging and in addition manual adjustment of residual low order aberrations further increases the dose at the sample.

Single shot coherent diffraction imaging (CDI) and ptychography has been widely used in light and Xray optics. The advantage of ptychography over traditional CDI is that it does not need prior information about the probe function and also overcomes some of the other issues of CDI, such as non-unique solutions and a limited field of view [2]. In electron microscopy, it has also attracted considerable interest due to its potential for super resolution [3], high phase sensitivity [5], three-dimensional [6] and low-dose [7] capabilities. One of the major developments that has advanced this field is the availability of a new generation of direct detection cameras that are particularly suited to ptychographic data acquisition with new modes of operation, such as electron counting and fast acquisition [8,9]. These new cameras dramatically also increase the detective quantum efficiency (DQE) and hence significantly improve the signal to noise ratio (SNR) of the recorded far field diffraction patterns (DP). Hence lower signals at higher scattering angles can be captured in each DP in a ptychograhic dataset which facilitates higher resolution in ptychographical reconstructions, even within the constraints of low electron dose work as required for beam sensitive samples.

In this paper, we will firstly review our previous work on the capabilities of defocused probe ptychography to achieve a 2D phase reconstruction of a nanocrystal at sub- $\AA$ resolution [4] and a 3D reconstruction of nanostructured materials [7] using traditional CCD camera. Subsequently we will show results from focused and defocused electron ptychography using a fast direct electron detector to reconstruct the wavefunction of various low dimensional materials under different low dose conditions. 
The low-dose experiment described were carried out on a JEM-ARM300F instrument [1] operated at $80 \mathrm{kV}$ with a Medipix3 direct electron detector [8]. Fig. 1a shows a schematic diagram of the optical configuration employed. A probe-forming convergence semi-angle of $24 \mathrm{mrad}$ was used and the sample was placed at a distance, $80 \mathrm{~nm}$ above the focal point as shown in Fig. 1a. The sample of monolayer $\mathrm{MoS}_{2}$ with an orientation along the $<001>$ zone axis, was illuminated with a probe in a $40 \times 40$ raster scan of probe positions and an approximate step size of $0.24 \mathrm{~nm}$ with a time interval of $2 \mathrm{~ms}$ [10].

References:

[1] H Sawada, et al., J Electron Microsc (Tokyo) 58 (2009), p. 357.

[2] AM Maiden and JM Rodenburg, Ultramicroscopy 109 (2009), p. 1256.

[3] PD Nellist, BC McCallum and JM Rodenburg, Nature 374 (1995), p. 630.

[4]P Wang, et al., Scientific Reports 7 (2017) , p.2857.

[5]H Yang, et al., Nat. Comm. 7 (2016) , p.12532.

[6] AJ D'Alfonso, et al., Journal of Applied Physics 119 (2016), p. 054302.

[7] S Gao, et al., Nature Communications, 8 (2017), p.163.

[8] JA Mir, et al., Ultramicroscopy, 182 (2017), p. 44.

[9] MW Tate, et al. Microsc. Microanal. 22 (2016), p. 237.

[10] The authors acknowledge funding from the National Natural Science Foundation of China (11474147), and the National Basic Research Program of China, (Grant No. 2015CB654901).

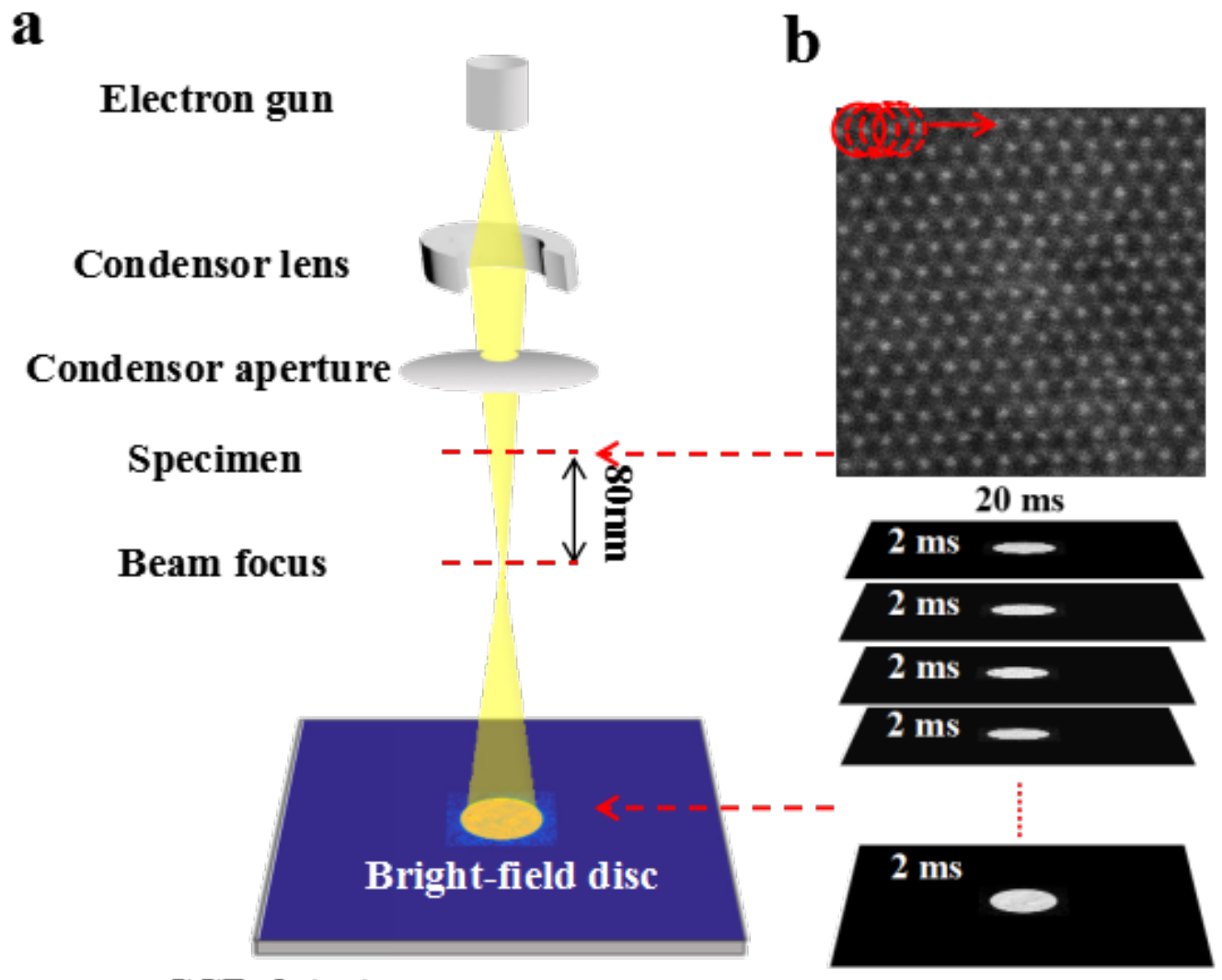

CCD detector

Figure 1. (a) Schematic of the experimental configuration used for ptychographic reconstruction. (b) HAADF image of a $\mathrm{MoS}_{2}$ monolayer oriented along a $<001>$ direction. 